\title{
Automatic Detection of Myocardial Boundaries in MR Cardio Perfusion Images
}

\author{
Luuk Spreeuwers $^{1}$ and Marcel Breeuwer ${ }^{2}$ \\ 1 University Medical Centre Utrecht, Image Sciences Institute, \\ luuk@isi.uu.nl \\ 2 Philips Medical Systems, \\ Medical Imaging Information Technology - Advanced Development, \\ marcel.breeuwer@philips.com
}

\section{Introduction}

Cardiovascular diseases often result in reduced blood perfusion of the myocardium (MC). Recent advances in MR allow fast recording of contrast enhanced myocardial perfusion scans. For perfusion analysis the myocardial boundaries must be traced. Currently this is done manually. In this paper a method for automatic detection of the myocardial boundaries is proposed.
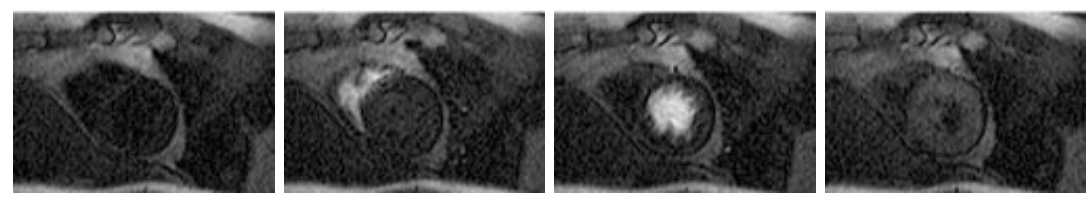

Fig. 1. Four images of a MRI perfusion data sequence; from left to right: no contrast, bolus passage in RV, bolus passage in LV, perfusion of the MC.

MR cardio perfusion scans are made by recording, during a period of 20-40 seconds, a number of short axis slices. The acquisition is controlled by the ECG so that each set of slices represents the same phase of the heart cycle. A few seconds after the beginning of the scan, contrast agent is injected. The contrast agent first enters the right ventricle (RV), then the left ventricle (LV) and finally the MC (see figure 1). The intensity as a function of time of a segment in the $\mathrm{LV}$ and $\mathrm{RV}$ and in the MC is sketched in figure 2a. The maximum upslope is most widely used [112] to characterise perfusion. To obtain the segments the boundaries of the $\mathrm{MC}$ have to be traced, see figure $2 \mathrm{~b}$.

\section{Myocardial Boundary Detection}

The most important consideration in the proposed approach is the use of both intensity-time and shape information to realise a robust segmentation. A block scheme of the myocardial boundary detection process is shown in figure 3 


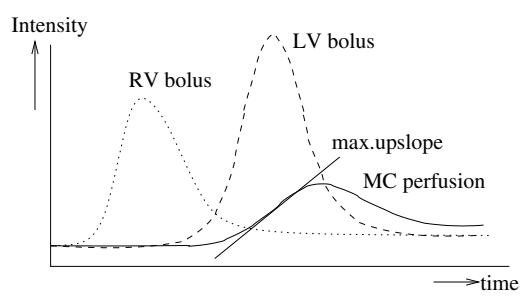

(a)

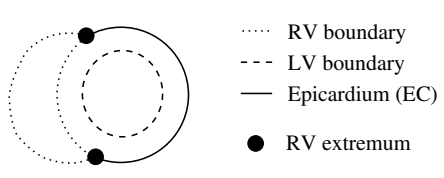

(b)

Fig. 2. a) Intensity-time profiles in the RV, LV and MC; b) boundaries to be traced.

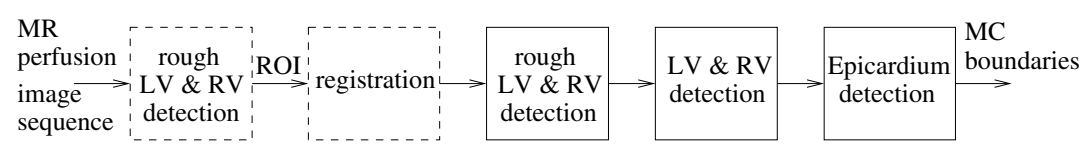

Fig. 3. Block scheme of the myocardial boundary detection process.

At bolus passage the LV and RV are very bright. Therefore, we search for very bright blobs in the image sequence. These blobs may be found by first smoothing in space and time to suppress smaller blobs caused by noise etc. and searching for the local maxima in space and time. Next feature images are constructed by correlating the time derivative of the intensity-time profiles of the RV and LV centres with those of all pixels in the image (figure 4 a).

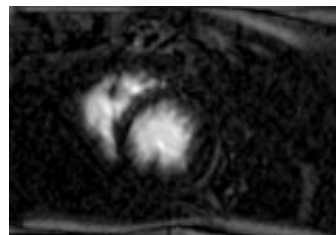

(a)

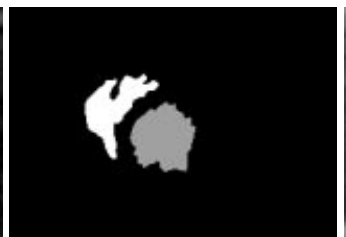

(b)

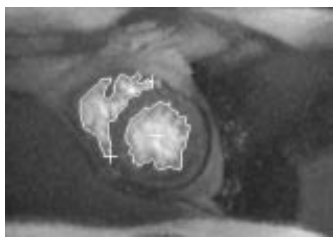

(c)

Fig. 4. LV and RV feature image (a), labelled regions (b) and boundaries (c).

The LV and RV are subsequently extracted by region growing from the RV and LV centres (figure $4 \mathrm{~b}$ ). The boundaries are extracted by tracing the outlines of the found regions. The extrema of the RV are detected by making a polar transform of the outline of the RV relative to the centre of gravity of the LV and searching for the extrema in the angles (figure 4c).

Detection of the EC is harder because the concentration of the contrast agent is much lower in the MC. Correlation of time-intensity curves did not result in usable features. To calculate a feature image for the MC, consider figure 5 

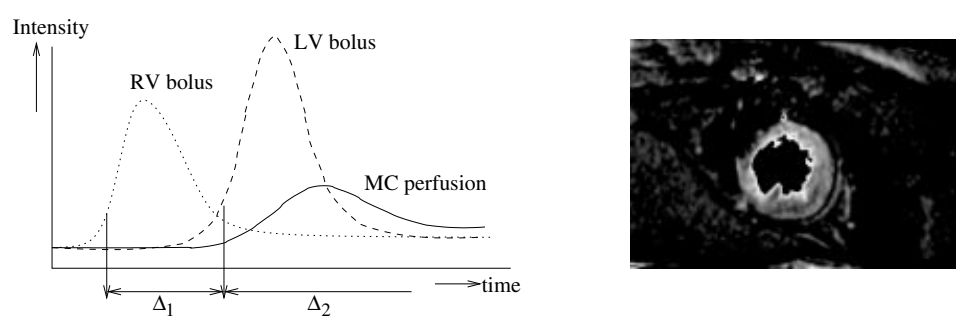

Fig. 5. MC feature image calculation. During $\Delta_{1}$ the $\mathrm{MC}$ is dark, during $\Delta_{2}$ the MC is bright. Subtracting MIPs of $\Delta_{1}$ and $\Delta_{2}$ gives an MC feature image (right).

The perfusion of the MC takes place during and after the bolus passage of the $\mathrm{LV}$. In period $\Delta_{1}$ there is no contrast agent in the MC and it is, therefore, dark. In period $\Delta_{2}$ the contrast agent enters the $\mathrm{MC}$ and it becomes brighter, while the intensity elsewhere remains the same. By subtracting a maximum intensity projection (MIP) of period $\Delta_{1}$ from a MIP of period $\Delta_{2}$, the MC shows up bright, while the surrounding tissues are suppressed. The resulting feature is shown on the right in figure 5 . The two RV extrema (figure 22) serve as starting points for the EC detection. Furthermore, it is assumed that the EC has a more or less circular shape. A polar transformation was generated from the $\mathrm{MC}$ feature image relative to the centre of gravity of the LV, see figure 6. Next a 5 node snake was fitted to the feature image, of which 2 , the RV extrema, were fixed (arrows in figure 6). the nodes is linear (in polar domain). The spline maximises the contrast ratio. Finally the boundary is transformed back into spatial domain.
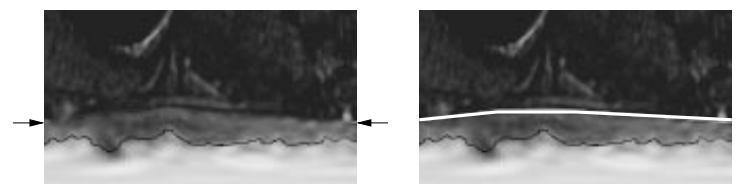

Fig. 6. Polar transformation (horizontal: angle, vertical: radius) of MC feature image (left). The RV-extrema are indicated by the arrows. Detected epicardium (right).

\section{Results}

The boundary detection procedure was tested on a total of 30 image sequences from 14 different scans. The scans varied in resolution $\left(128^{*} 128\right.$ and $256^{*} 256$ pixels) and number of slices (1-5). The detected boundaries were evaluated by visual inspection. From 26 out of 30 sequences the myocardial boundaries were 
found correctly. The four remaining sequences contained severe motion artifacts. Segmentation of a $256^{*} 256^{*} 70$ sequence took about 25 seconds on a Sun UltraSPARC-IIi @440 MHz. Figure 7 shows some results.
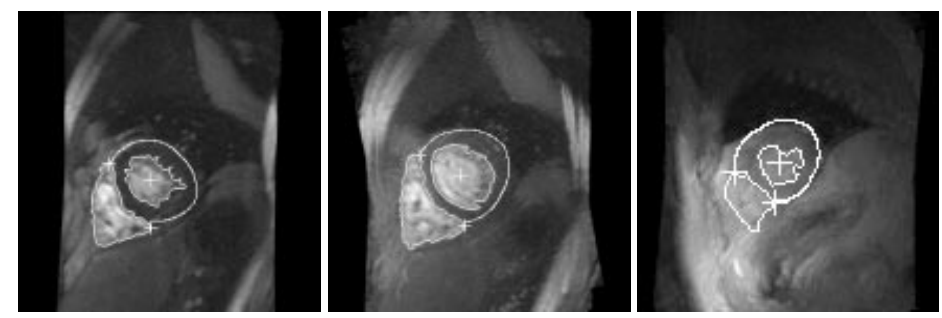

Fig. 7. Some results of the boundary detection method for different types of images

\section{References}

1. N. Al-Saadi, E. Nagel, M. Gross, A. Bornstedt, B. Schnackenburg, C. Klein, H. Oswald, and E. Fleck, "Non-invasive detection of myocardial ischemia from perfusion reserve based on cardiovascular magnetic resonance," in Circulation, No. 101, 2000. 1st Virtual Congress of Cardiology, http://pcvc.sminter.com.ar/cvirtual.

2. M. Breeuwer, L. Spreeuwers, and M. Quist, "Automatic quantitative analysis of cardiac MR perfusion images," in Proceedings of SPIE Medical Imaging, (San Diego, USA), February 2001. 